\title{
Determination of ochratoxin A in licorice root using inverse ion mobility spectrometry
}

\author{
Mohammadreza Khalesi ${ }^{a}$, Mahmoud Sheikh-Zeinoddin ${ }^{\mathrm{a}}$, Mahmoud Tabrizchi ${ }^{\mathrm{b}, *}$ \\ a Department of Food Science and Technology, College of Agriculture, Isfahan University of Technology, Isfahan, 84156-83111, Iran \\ b Department of Chemistry, Isfahan University of Technology, Isfahan, 84156-83111, Iran
}

\section{A R T I C L E I N F O}

\section{Article history:}

Received 14 August 2010

Received in revised form 30 October 2010

Accepted 1 November 2010

Available online 10 November 2010

\section{Keywords:}

Ochratoxin A

Ion mobility spectrometry

Licorice

\begin{abstract}
A B S T R A C T
Despite the recent, successful efforts to detect mycotoxins, new methods are still required to achieve higher sensitivity, more simplicity, higher speed, and higher accuracy at lower costs. This paper describes the determination of ochratoxin A (OTA) using corona discharge ion mobility spectrometry (IMS) in the licorice root. A quick screening and measuring method is proposed to be employed after cleaning up the extracted OTA by immunoaffinity columns. The ion mobility spectrometer is used in the inverse mode to better differentiate the OTA peak from the neighboring ones. After optimization of the experimental conditions such as corona voltage, injection port temperature, and IMS cell temperature, a limit of detection (LOD) of $0.010 \mathrm{ng}$ is obtained. Furthermore, the calibration curve is found to be in the range of $0.01-1 \mathrm{ng}$ with a correlation coefficient $\left(R^{2}\right)$ of 0.988 . Licorice roots were analyzed for their OTA content to demonstrate the capability of the proposed method in the quantitative detection of OTA in real samples.
\end{abstract} (C) 2010 Elsevier B.V. All rights reserved.

\section{Introduction}

Mycotoxins are toxic products formed as secondary metabolites by some fungal species that readily colonize and contaminate both field and harvested crops with toxins [1,2]. Ochratoxins are a group of mycotoxins produced by two genera of fungi: Penicillium and Aspergillus. Ochratoxin A (OTA) is the most toxic member of the group, which was first isolated from Aspergillus ochraceus Wilh. [3] and has been classified as a nephrotoxic, hepatotoxic, teratogenic, and immunotoxic compound. It is also reasonably predicted to be a possible human carcinogen due to adequate evidence of carcinogenicity in experimental animals (group 2B) [4]. Its empirical formula is $\mathrm{C}_{20} \mathrm{H}_{18} \mathrm{O}_{6} \mathrm{NCl}$ and its molecular weight is $403.82 \mathrm{~g} \mathrm{~mol}^{-1}$ [5]. Fig. 1 shows the OTA structure.

The fact that most mycotoxins are toxic at low concentrations requires sensitive and reliable methods for quantitative detection. Sampling and analysis are of critical importance since failure to attain an agreeable verified analysis can lead to ineligible consignments being accepted, or satisfactory loads being unnecessarily rejected [6]. Several analytical techniques, which offer flexible analysis and, in some cases, yield satisfactory OTA detection, have been discussed in a review by Turner et al. [7]. Many of these methods are lab-based, but there seems to be no distinct technique that stands out above the rest, even though analytical liquid chromatography

\footnotetext{
* Corresponding author. Tel.: +98 311 3913272; fax: +98 3113912350.

E-mail address: m-tabriz@cc.iut.ac.ir (M. Tabrizchi).
}

commonly coupled with mass spectroscopy is likely to be admired. Some manuscripts discuss (i) sample pretreatment methods such as liquid-liquid extraction (LLE) [8], and solid phase extraction (SPE) [9]; (ii) separation methods such as gas chromatography (GC) [10], thin layer chromatography (TLC) [11], high performance liquid chromatography (HPLC) [12,13], and capillary electrophoresis (CE) [14]; and (iii) others such as ELISA [15] as reputed methods. Almost all advantages, disadvantages and future prospects of these methods have been discussed in Turner et al. [7].

Licorice (Glycyrrhiza glabra L., Leguminosae), a crop that can easily be contaminated with OTA, is a common dietary supplement for its pharmaceutical properties, the most important ones being that it can both act as an expectorant and raise the blood pressure. It is used as an ingredient for herbal infusions in the form of licorice root [16]. Licorice derivatives are largely consumed as flavoring and sweetening agents in confectionery and other food products, such as beverages and chewing gums [17-19]. Licorice products are also used in cigarettes both as a flavor and a casing agent [18]. Although there is a lack of information on the OTA contamination of certain minor food commodities such as licorice, spices, and teas [20], there is COMMISSION REGULATION (EU) no. $105 / 2010$ amending regulation (EC) no. 1881/2006 setting maximum levels for certain contaminants in foodstuffs. The following maximum levels are proposed with regard to ochratoxin A: licorice root $20 \mu \mathrm{g} / \mathrm{kg}$ and licorice extract $80 \mu \mathrm{g} / \mathrm{kg}$ [21].

A successful method for the detection and determination of OTA in licorice and many other crops should be robust and sensitive with a high degree of flexibility over a large range of compounds, 


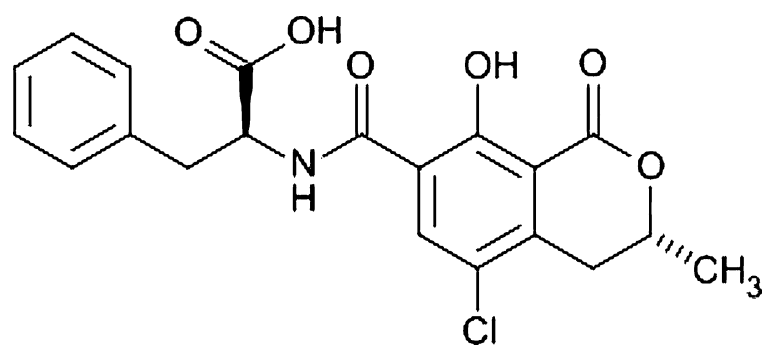

Fig. 1. Chemical structure of OTA.

but which can be specific enough when required [7]. The technique should also be easy to use, rapid, and portable, especially to suit the conditions in processing plants where tons of quality approved licorice root should be obtained from different suppliers and stored for a relatively long period of time before processing.

IMS, which is basically a gas-phase ion separation technique, has achieved wide acceptance in many applications for detecting contaminants due to its excellent sensitivity and quick operation. A full description of the technique is provided in several books and review articles [22,23]. Low detection limit, fast response, simplicity, portability, and relatively low cost are its main advantages. The technique is similar to time of flight mass spectrometry except that it runs under atmospheric pressure. An extensive range of compounds such as explosives [24], narcotics [25], drugs of abuse [26], herbicides [27], pesticides [28], and aflatoxin B [29] have been detected by IMS. The ionization scheme in IMS is based on protonation of compounds. Due to its chemical structure, OTA is expected to be easily protonated in IMS. Hence, IMS could be a potentially excellent technique for OTA determination.

The objective of the present study is to evaluate the performance of IMS in the determination of ochratoxin A in licorice roots. Measurements were carried out in the inverse operation mode to enhance the separation power of ion mobility spectrometry [30]. This novel method was developed in our lab in which, rather than generating an ion packet, a dip was created in the ion beam, hence increased resolution by up to $60 \%$ as compared to the conventional linear mode.

\section{Experimental}

\subsection{Ion mobility spectrometer}

Fig. 2 shows the schematic diagram of the ion mobility spectrometer used in this study. It was constructed in our laboratory at Isfahan University of Technology. The IMS cell, consisting of the ionization and drift regions, was housed in a thermostatic oven where the temperature could be adjusted from room temperature to $200{ }^{\circ} \mathrm{C}$. The ionization region consisted of five aluminum rings $0.95 \mathrm{~cm}$ thick, $20 \mathrm{~mm}$ ID and $55 \mathrm{~mm}$ OD. The drift tube also consisted of 11 similar aluminum rings $36 \mathrm{~mm}$ ID. Thin Teflon insulators were inserted between the rings. Each ring was connected to the adjacent one via a $5 \mathrm{M} \Omega$ resistor to create a potential gradient. A voltage of $7 \mathrm{kV}$ was applied over the entire cell to create a drift field of $437.5 \mathrm{~V} / \mathrm{cm}$. The instrument was equipped with a needleto-plane corona discharge ionization source described by Tabrizchi et al. [31]. The needle voltage was independently adjustable. In all the experiments, the needle potential was kept constant to create a stable and steady corona.

A Bradbury-Nielsen type gate [32] was mounted between the ionization region and the drift tube. The gate consisted of two sets of parallel wires mounted on a ceramic frame in a plane perpendicular to the moving direction of ions. When an adequate potential was applied to the wires, the electric field created between the wires
Table 1

The optimized experimental conditions for OTA determination.

\begin{tabular}{ll}
\hline Parameter & Setting \\
\hline Length of drift tube & $11 \mathrm{~cm}$ \\
Drift field & $437.5 \mathrm{~V} \mathrm{~cm}^{-1}$ \\
Drift voltage & $7000 \mathrm{~V}$ \\
Corona voltage & $2300 \mathrm{~V}$ \\
Flow of drift gas $\left(\mathrm{N}_{2}\right)$ & $700 \mathrm{~mL} \mathrm{~min}^{-1}$ \\
Flow of carrier gas $\left(\mathrm{N}_{2}\right)$ & $300 \mathrm{~mL} \mathrm{~min}^{-1}$ \\
Injection port temperature & $260^{\circ} \mathrm{C}$ \\
IMS cell temperature & $200^{\circ} \mathrm{C}$ \\
\hline
\end{tabular}

prevented ion penetration; hence, the gate was closed. Conversely, the gate opened when the potential was removed. A lab-made pulse generator was used to apply a pulsed voltage to the shutter grid. The gate was usually closed except for a short period of time when an ion packet was to be injected into the drift region. However, the pulse generator was designed to have the inverse option for the pulse; therefore, the gate was normally kept open except for the short time when it was closed. In this way, a dip was created in the continuous current of ions within the drift region. The dip moved at the same velocity as the ion packet, and the detector read an inverse peak at a drift time similar to that of conventional linear mode of operation. This new technique, named inverse ion mobility spectrometry, was recently invented in our lab to enhance IMS resolution [30]. Except for the optimization, all the measurements including the calibration curve were obtained in the inverse mode.

An analog to digital A/D converter (PicoScope, UK) was used and the digitized signal was averaged over a number of scans. The resulting ion mobility spectrum was then displayed on the monitor. Nitrogen gas, after passing through a $13 \times$ molecular sieve (Fluka), was passed through the cell at 300 and $700 \mathrm{~mL} \mathrm{~min}^{-1}$ as the carrier and drift gases, respectively. The spectrometer was operated in the positive mode. Table 1 presents the optimized experimental conditions for obtaining ion mobility spectra of OTA.

\subsection{Other apparatus and reagents}

The IKA ULTRA-TURRAX ${ }^{\circledR}$ T 25 digital was used for homogenizing the samples. A laboratory centrifuge (SiGMA 6K15) was used to separate soluble from insoluble materials. Immunoaffinity columns (Puri-Fast ${ }^{\circledR}$ OTA IAC) were also purchased from Libios Co., France.

Reagents for sodium bicarbonate $\mathrm{pH} 8.1(0.13 \mathrm{M})$ and phosphate buffer saline (PBS) pH 7.4 (20 mM) were prepared using pure materials from Merck, Germany.

Methanol was chosen as the solvent because it possesses a much lower proton affinity than the analyte. This allows the ionization of the analyte in the presence of large amounts of the solvent. It generates a very short-lived peak close to the reactant ion peak (RIP) which has no interference with that of OTA. Analysis of OTA revealed that the use of methanol as an extraction solvent for OTA offers such advantages as high recoveries and lower costs while it is more environmental friendly as well $[33,34]$. Ochratoxin A standard was purchased from Sigma (St. Louis, MO) and stock solutions (0.01 $\left.\mathrm{mg} \mathrm{mL}^{-1}\right)$ were prepared in methanol $(99.5 \%(\mathrm{v} / \mathrm{v})$, Merck) and stored at $-21^{\circ} \mathrm{C}$.

\subsection{Samples}

A total of $6 \mathrm{~kg}$ licorice root was supplied by Rishmac Co. from Beiza region, Fars Province, Iran, and delivered by road in less than $8 \mathrm{~h}$ to the Food Microbiology Laboratory at Isfahan University of Technology where it was prepared for analysis. 


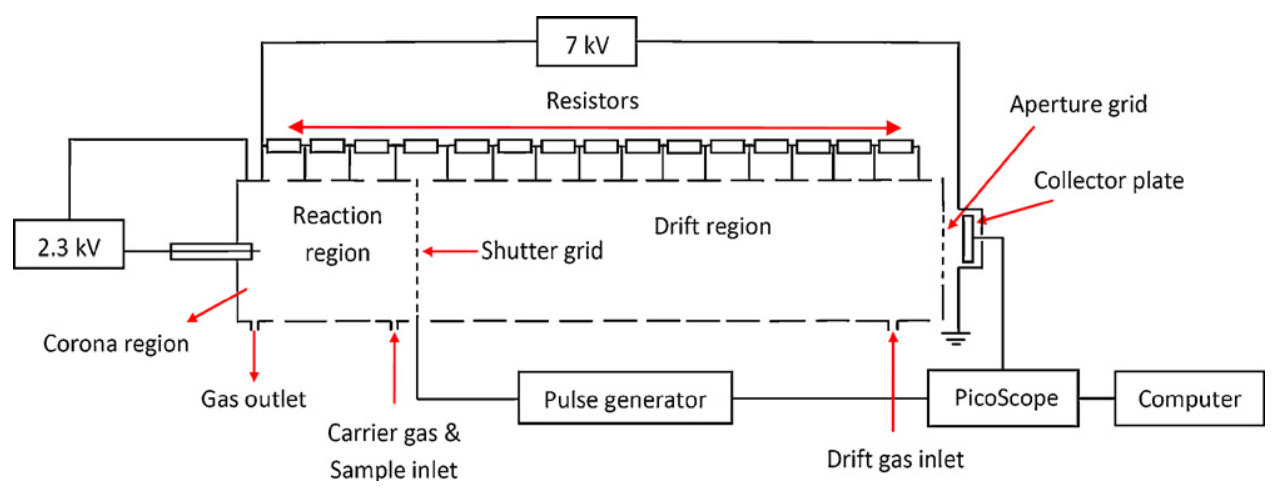

Fig. 2. The schematic diagram of the ion mobility spectrometer.

\subsection{Ochratoxin A extraction and purification}

OTA extraction was carried out when needed according to the method described by Herrera et al. [16]. Briefly, $3 \mathrm{~g}$ of ground licorice root powder was added to a mixture of $30 \mathrm{~mL}$ sodium bicarbonate $0.13 \mathrm{M}$ and methanol (9:1,v/v) which was then homogenized in an Ultrat-turrax homogenizer for $2 \mathrm{~min}$. The extract was then centrifuged at $3500 \mathrm{rpm}$ (radius $15 \mathrm{~mm}$ ) for $15 \mathrm{~min}$. The supernatant $(10 \mathrm{~mL})$ was purified using an immunoaffinity column pre-washed by $10 \mathrm{~mL}$ PBS ( $\mathrm{pH} 7.4$ ). The purified extract was then eluted slowly (one drop per second.) through the column. The OTA trapped in the column was recovered using $10 \mathrm{~mL}$ of methanol/acetic acid (98:2, v/v) used as the solvent. To concentrate the recovered OTA, the solvent was completely evaporated under a gentle stream of dry nitrogen at $30^{\circ} \mathrm{C}$, and the remaining sample was re-dissolved in $1 \mathrm{~mL}$ methanol. Finally, aliquots of $10 \mu \mathrm{L}$ were injected into the ion mobility spectrometry for analysis.

\section{Results and discussion}

\subsection{Ion mobility spectrum}

The ion mobility spectrum of OTA at $200^{\circ} \mathrm{C}$ is given in Fig. 3 . At high concentrations ( $100 \mathrm{ng}$ OTA) the spectrum contains several broad peaks from 6 to $12 \mathrm{~ms}$. Although precise assignment of the peaks needs a mass spectrometer coupled to the IMS, it could be speculated that, due to the high molecular weight, the last peak of around $11.44 \mathrm{~ms}$ originated from the protonated OTA, the rest

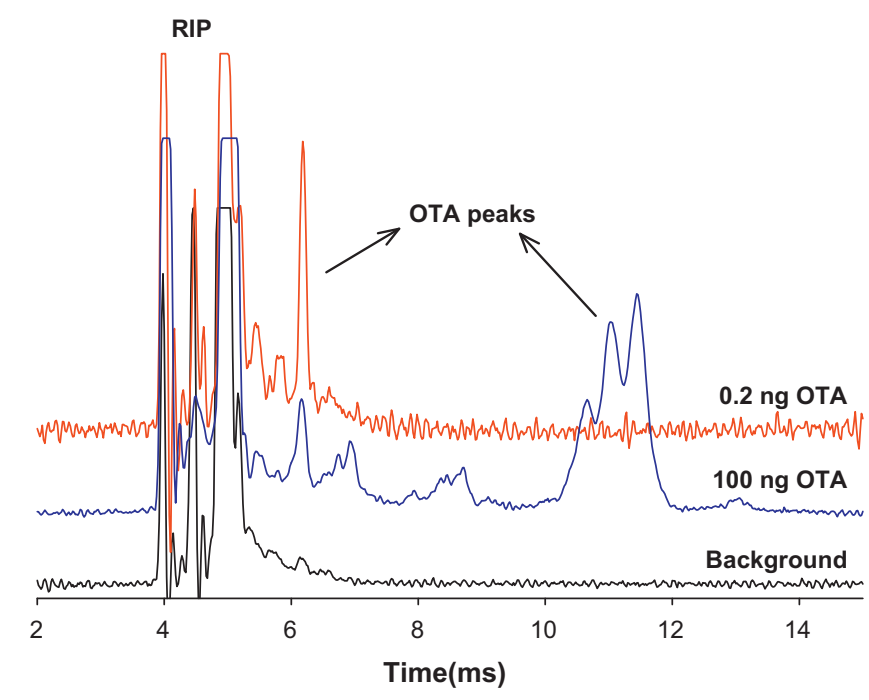

Fig. 3. Ion mobility spectrum of OTA at $200^{\circ} \mathrm{C}$. being its fragments. It has been shown that OTA starts decomposition at around $100{ }^{\circ} \mathrm{C}$ with a relatively long life [35]. It should be mentioned that peaks of even low OTA concentrations remain stable for a relatively long time in the IMS spectrum. For example, the spectrum shown in Fig. 3 for 100 ng OTA was recorded one hour after the injection had been made. The half-life for OTA decomposition is reported to be $12 \mathrm{~min}$ at $200^{\circ} \mathrm{C}$ under dry conditions [35]. Thus, $3 \%$ of the original OTA, equivalent to $3 \mathrm{ng}$, was still observed after one hour. Such low amounts of OTA are detectable by this technique in Fig. 3, the component peaks of OTA are observable for a still lower amount of $0.2 \mathrm{ng}$.

The ion mobility spectrum of OTA at lower concentrations contains only the fragment peak at $6.18 \mathrm{~ms}$. An example is demonstrated in Fig. 3 (the upper trace) for $0.2 \mathrm{ng}$ OTA in $10 \mu \mathrm{L}$ methanol. The narrow peak at $6.18 \mathrm{~ms}$ has the longest life time among all the fragments. Therefore, it was chosen as an indicator of OTA and its height was integrated over the acquisition time and taken as the response of the IMS to the analyte. Other peaks appearing at higher concentrations were neglected since in contaminated food products, OTA concentration does not normally exceed this range [36].

Although the narrow peak appears near the reactant ion peak, in a region where also lie the drift times of many other compounds, its long life time is an excellent indicator for discriminating between OTA and other compounds. On the other hand, the product ion peak in IMS in most cases disappears in less than one minute depending on OTA concentration. Therefore, integration of the peak over a long acquisition time minimizes interference from other compounds, even though their peak may appear in the same region as the OTA peak does. In addition, the long life of the peak generates a large signal since the signal is recorded as the volume under the OTA peak in the two dimensions of drift time and acquisition time, which results in a low detection limit. In conclusion, the long life time of OTA is a great advantage not only for discriminating OTA peak but also for detecting this hazardous mycotoxin at trace levels.

\subsection{Optimization}

The ion mobility spectra of OTA were obtained under the optimized experimental conditions given in Table 1.

The injection port temperature was optimized by evaluating the signal intensity for $0.2 \mathrm{ng}$ OTA at different temperatures. The optimized injection temperature varies with the structure of the compound, its stability, and its melting point. In the case of OTA, when temperature goes up, the signal intensity increases as shown in Fig. 4. This could be due to the increased decomposition rate of OTA at higher temperatures. The highest possible temperature (i.e. $260^{\circ} \mathrm{C}$ ) was chosen for the injection port. Such a high temperature is quite appropriate for quick evaporation of the methanol solvent as well. 


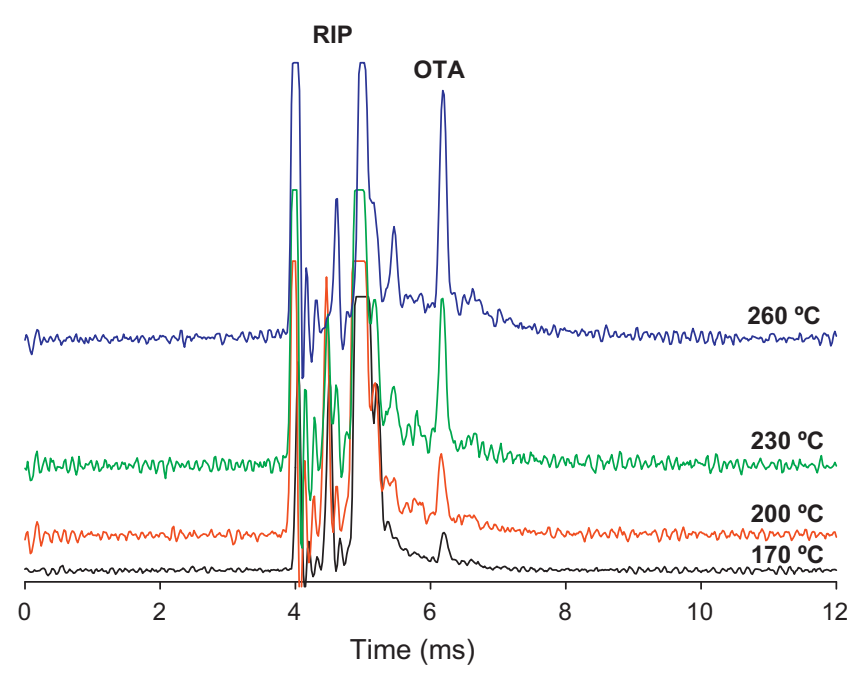

Fig. 4. The effect of injection temperature on the intensity of OTA peak.

Similarly, the best temperature for the IMS cell was found to be the highest available value, i.e. $200^{\circ} \mathrm{C}$. This is high enough to prevent solvent condensation inside the cell. It also helps dehydration of ions, resulting in a better resolution [37]. At lower temperatures, the memory effect was a real challenge. In addition, the high temperature of the cell temperature prevented long memory effects.

\subsection{The inverse mode}

As described earlier, the OTA peak appears in a region where those of most other compounds do. It was noticed in real sample analysis that the OTA peak was partially masked by a nearby broad peak due to unknown substances present in the licorice root. This is shown in Fig. 5 (the upper trace). The small OTA peak was surrounded by background peaks and, therefore, its integration proved difficult. It has been previously shown that resolution consider-

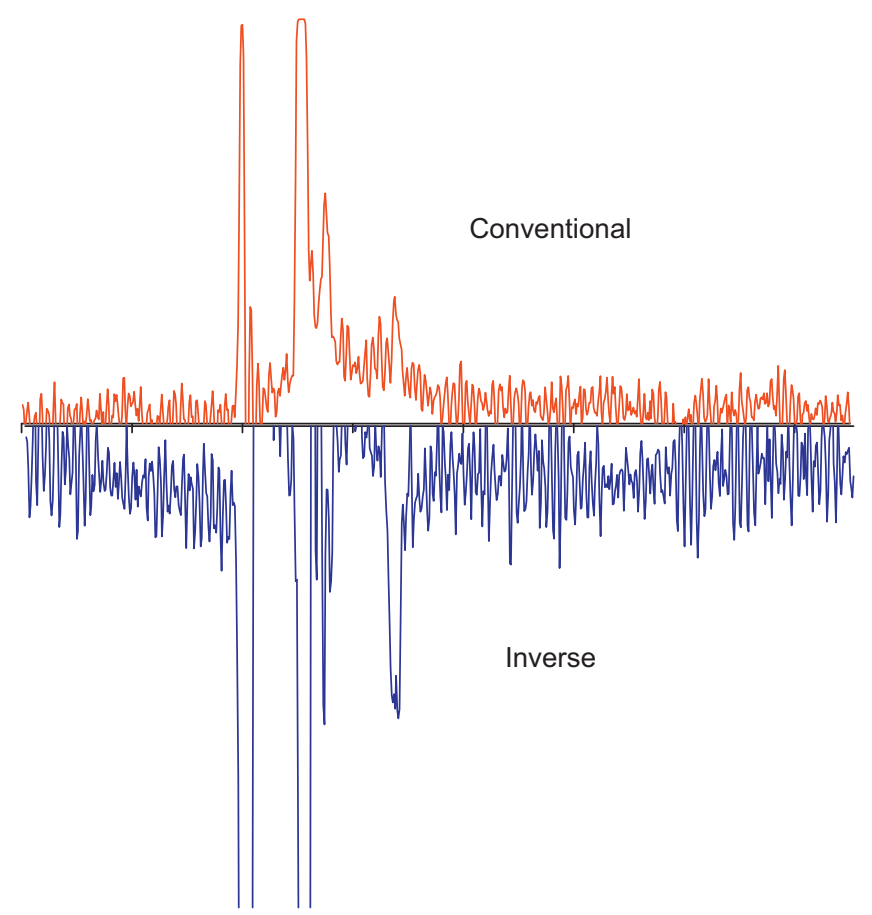

Fig. 5. A single run ion mobility spectrum of the extracted OTA in conventional linear and inverse mode.

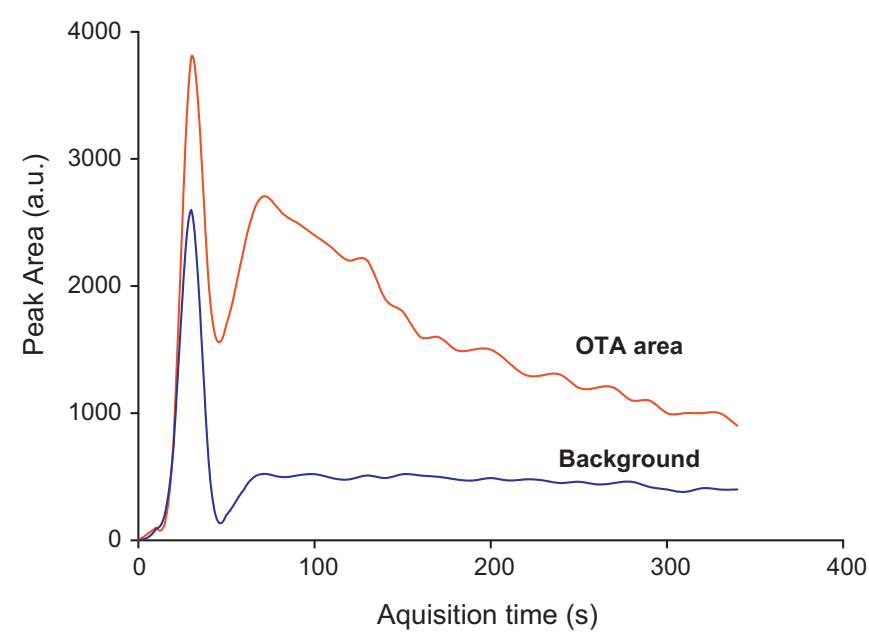

Fig. 6. Time evolution of the OTA peak for $0.2 \mathrm{ng}$ OTA as well as that of the background. The area between the two curves was chosen for the signal.

ably increases in the inverse mode [30]. Hence, the technique was applied in order to better differentiate between the OTA peak and its busy surrounding. The result is shown in Fig. 5 (the lower trace), where the inverse spectrum is compared with the conventional linear one. Clearly, the OTA peak in the inverse mode is more clearly recognized from its surrounding peaks. The reason for the better resolution in the inverse mode is believed to be the absence of space charges within the dips [38]. As the ion density in our instrument is high, the space charge is an important cause of broadening in the conventional linear mode [38]. In the inverse mode, dips travel through a bath of ions and ion density inside the dip is lower than that on either side of the dip. Hence, the space charge is in favor of narrowing the dip. For consistency in the results obtained, not only was the calibration curve prepared in the inverse mode but also all the measurements were performed in the same mode.

\subsection{Time evolution}

In practice, when $10 \mu \mathrm{L}$ of blank methanol was injected into the port, the corona discharge was shortly disturbed. This was evident by a quick change in the baseline of the spectrum that was then quickly stabilized. When the solvent contained a small amount of OTA, after the baseline had been disturbed and restabilized again, the OTA peak rose to its maximum level when it began to decay at an exponential rate. This trend is shown in Fig. 6 where the background trend is also shown. The large peak at the beginning reflects the disturbance in the background. The background signal was obtained from the integration of the same window as that used for the analyte peak in its neighborhood.

\subsection{Calibration curve}

The calibration curve was obtained by calculating the area between the two curves in Fig. 6 for 300 s starting from the injection time. A similar procedure was used for different concentrations and for the analysis of real samples. For each concentration, a $10 \mu \mathrm{L}$ standard solution was injected into the injection port. As depicted in Fig. 7, the calibration curve is almost linear over $0.01-1 \mathrm{ng}$ OTA. Hence, a two orders of magnitude dynamic range was achieved. The practical detection limit, LOD, was obtained to be about $10 \mathrm{pg}$ based on a signal to noise ratio of $3: 1$. This is comparable with those reported for other qualified techniques $[15,16]$. 


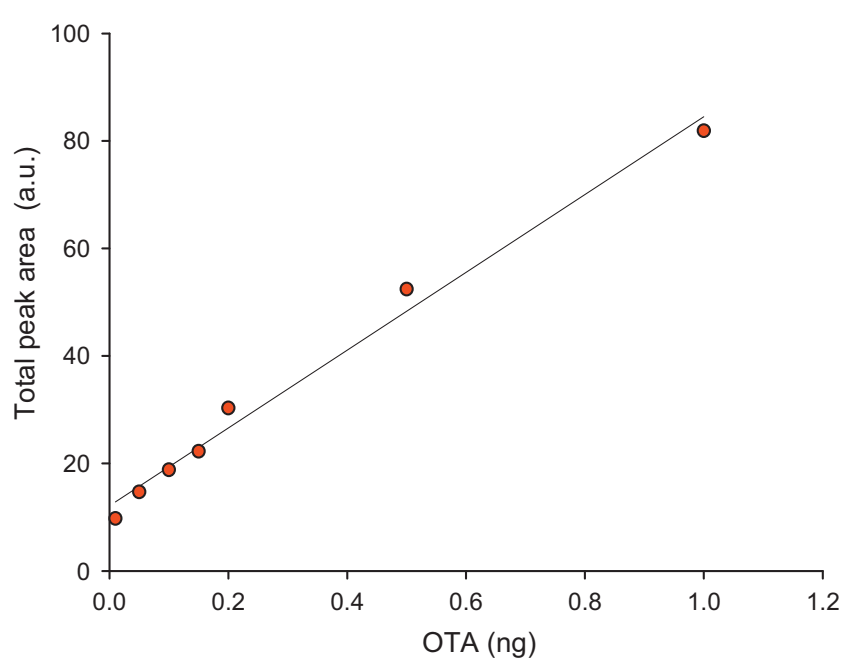

Fig. 7. The calibration curve for OTA determination.

\subsection{Analysis of real samples}

Licorice root was chosen as the real sample. It was divided into two halves, one sent to an accredited lab, Gesellschaft für Bioanalytik Hamburg, Germany (GBA), and one was used for extraction using the procedure described in the experimental part above. $10 \mu \mathrm{L}$ of the extracted OTA was then injected into the IMS. The ion mobility spectrum shown in Fig. 8 (the upper trace) contains a large peak at $10.78 \mathrm{~ms}$ which chemically masks the OTA peak. This peak could belong to glycyrrhizic acid present in the licorice root in grate abundance [39].

In order to extract OTA more selectively, the immunoaffinity column was used. For this purpose, the ion mobility spectrum of the extracted OTA after cleaning up using immunoaffinity columns is given in Fig. 8 (the lower trace). All unwanted peaks were eliminated while the OTA peak was distinctly present.

The recovery was determined at three levels $(20,50$, and $100 \mathrm{ng} / \mathrm{g}$ ) in triplicates by spiking blank licorice sample with OTA. The recoveries resulted in 111,109 , and $111 \%$, respectively, with about $1 \%$ relative standard deviation (RSD\%).

The amount of OTA in the real sample corresponding to Fig. 8 was calculated to be $88 \pm 6 \mathrm{pg} / \mathrm{g}$ equivalent to $8.8 \pm 0.6 \mathrm{ng} / \mathrm{g}$ licorice root after recovery corrections were affected. This was very close to the value $(8.5 \mathrm{ng} / \mathrm{g})$ reported by GBA Lab, Germany, for a similar sample. To verify the results, a similar procedure was used for a

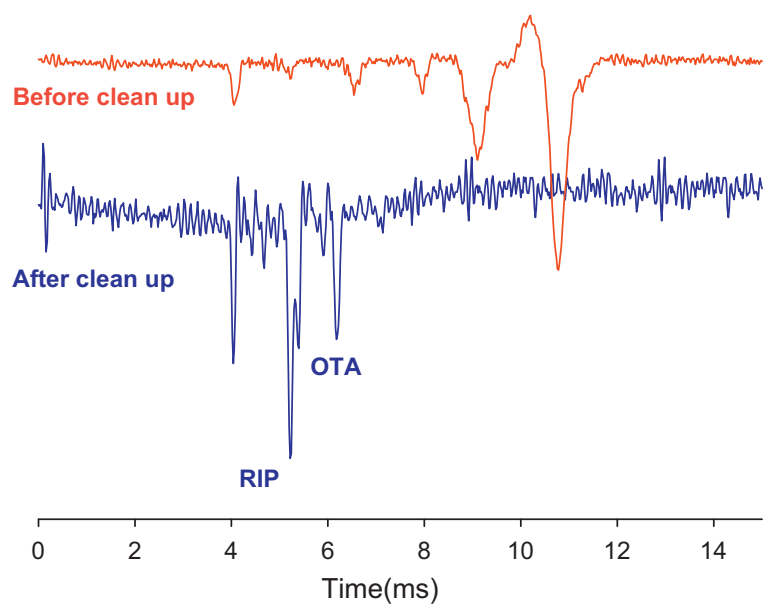

Fig. 8. Spectra of extracted OTA in licorice root before and after cleaning up by IAC. second fresh sample of licorice root and the OTA content was found to be $0.6 \pm 0.1 \mathrm{ng} / \mathrm{g}$, which is comparable to $0.5 \mathrm{ng} / \mathrm{g}$ reported by GBA Lab.

\section{Conclusion}

This study demonstrates the capability of positive corona discharge inverse ion mobility spectrometry for quantitative determination of OTA in licorice root as a real sample. This novel approach yields an acceptable detection limit, a good accuracy, and an appropriate recovery level. The detection limit obtained by this technique is comparable to that reported by others. However, the other methods are generally expensive and time-consuming. Fast response, low cost, simplicity, and portability of the IMS can make the method competitive among the techniques used to detect and determine ochratoxin $A$ in crops like licorice root that are susceptible to contamination by fungi. The nature of licorice product makes this technique a potentially vital part of their quality control. Licorice root is normally stocked in extraction plant in large quantities for long storage times (even up to one year) before they are processed. For such plants which are typically cultivated and used in less developed countries, it is much easier to control their contamination by an easy-to-use and inexpensive technique such as inverse IMS.

The new method is valid for screening purposes and reliable if the extract is subjected to a previous immunoaffinity cleanup. However, it is simple enough to be applied in countries of origin known to be the major producer of licorice products. Due to complexity of the current available techniques, only a few laboratories, mainly in Europe and North America, are known to carry out such analysis. Hence, it usually takes a few weeks for a sample to be sent to the credible lab and analyzed. Considering these facts, the application of IMS in measuring OTA could be regarded as a rather faster, more available method.

\section{Acknowledgements}

The authors would like to appreciate Rishmac Co. for funding this research project and staff of both Food Biotechnology and IMS lab, Isfahan University of Technology, for their cooperation. The Center of Excellency for Sensor and Green Chemistry of IUT also deserves our gratitude for its support.

\section{References}

[1] J.M. Wagacha, J.W. Muthomi, Int. J. Food Microbiol. 124 (2008) 1.

[2] G. Gonzalez, M.J. Hinojo, R. Mateo, A. Medina, M. Jimenez, Int. J. Food Microbiol. 105 (2005) 1.

[3] D. Ringot, A. Chango, Y.J. Schneider, L.Y. Yvan, Chem. Biol. Interact. 159 (2006) 18.

[4] IARC, Agents reviewed by the IARC monographs, vols. 1-100a (by cas numbers) (2009) P:10.

[5] B. Huang, W. Tao, J. Shi, L. Tang, J. Jin, Arch. Toxicol. 80 (2006) 481.

[6] D.R. Lauren, D.J. Jensen, W.A. Smith, N.Z. J. Crop Hortic. 34 (2006) 63.

[7] N.W. Turner, S. Subrahmanyam, S.A. Piletsky, Anal. Chim. Acta 632 (2009) 168

[8] L. Monaci, G. Tantillo, F. Palmisano, Anal. Bioanal. Chem. 378 (2004) 1777.

[9] M. Vega, K. Munoz, C. Sepulveda, A. Mario, V. Campos, R. Villegas, O. Villarroel, Food Control 20 (2009) 631

[10] G.J. Soleas, J. Yan, D.M. Goldberg, J. Agric. Food Chem. 49 (2001) 2733.

[11] M. Yamazaki, Y. Maebayashi, K. Miyaki, Appl. Microbiol. 20 (3) (1970) 452.

[12] C. Zaied, S. Abid, L. Zorgui, C. Bouaziz, S. Chouchane, M. Jomaa, H. Bacha, Food Control 20 (2009) 218

[13] A. Gallo, G. Perrone, M. Solfrizzo, F. Epifani, A. Abbas, A.D.W. Dobson, G. Mule Int. J. Food Microbiol. 129 (2009) 8

[14] E. Gonzalez-Penas, C. Leache, A.L. de Cerain, E. Lizarraga, Food Chem. 97 (2006) 349.

[15] S.H. Alarcon, G. Palleschi, D. Compagnone, M. Pascale, A. Visconti, I. Barna-Vetro, Talanta 69 (2006) 1031.

[16] M. Herrera, A. Herrera, A. Arino, Food Chem. Toxicol. 47 (2009) 2002.

[17] D. Gabriele, S. Curcio, B. Cindio, J. Food Eng. 48 (2009) 33.

[18] E.L. Carmines, R. Lemus, C.L. Gaworsky, Food Chem. Toxicol. 43 (2005) 1303 
[19] A.M. Arino, M. Herrera, G.T. Estopanan, T. Juan, Int. J. Food Microbiol. 114(2007) 366.

[20] J. Fink-Gremmels, Food Addit. Contam. 22 (Suppl. 1) (2005) 1.

[21] COMMISSION REGULATION (EU) No 105/2010 of 5 February 2010 amending Regulation (EC) No 1881/2006 setting maximum levels for certain contaminants in foodstuffs as regards ochratoxin A. Off. J. Eur. Union 6.2.2010.

[22] G.A. Eiceman, Z. Karpas, Ion Mobility Spectrometry, CRC Press, Boca Raton, FL, 2005.

[23] C.S. Creaser, J.R. Griffiths, C.J. Bramwell, S. Noreen, C.A. Hill, C.L.P. Thomas, Analyst 129 (2004) 984.

[24] M. Tabrizchi, V. ILbeigi, J. Hazard. Mater. 176 (2010) 692.

[25] T. Khayamian, M. Tabrizchi, M.T. Jafari, Talanta 69 (2006) 795.

[26] T. Keller, A. Miki, P. Regenscheit, R. Dirnhofer, A. Schneider, H. Tsuchihashi, Forensic Sci. Int. 94 (1998) 55.

[27] B.H. Clowers, W.E. Steiner, H.M. Dion, L.M. Matz, M. Tam, E.E. Tarver, H.H. Hill, Field Anal. Chem. Technol. 5 (6) (2001) 302.
[28] K. Tuovinen, H. Paakkanen, O. Hanninen, Anal. Chim. Acta 404 (2000) 7.

[29] A. Sheibani, M. Tabrizchi, H.S. Ghaziaskar, Talanta 75 (2008) 233.

[30] M. Tabrizchi, E. Jazan, Anal. Chem. 82 (2010) 746.

[31] M. Tabrizchi, T. Khayamian, N. Taj, Rev. Sci. Instrum. 71 (6) (2000) 2321.

[32] N.E. Bradbury, R.A. Nielsen, Phys. Rev. 49 (1936) 388.

[33] L.G. Osnaya, J.M.S. del Castillo, J.C.M. Cortes, J.M. Vinuesa, J. Chromatogr. A 1113 (2006) 32.

[34] C. Juan, L. GonzaLez, J.M. Soriano, J.C. Molto, J. Manes, J. Agric. Food. Chem. 53 (2005) 9348.

[35] H. Boudra, P.L. Bars, J.L. Bars, Appl. Environ. Microbiol. 61 (3) (1995) 1156.

[36] C. Brera, R. Caputi, M. Miraglia, I. Iavicoli, A. Salerno, G. Carelli, Microchem. J. 73 (2002) 167.

[37] M. Tabrizchi, Talanta 62 (2004) 65.

[38] G.E. Spangler, Anal. Chem. 82 (2010) 8052.

[39] J.R. Hennell, S. Lee, C.S. Khoo, M.J. Gray, A. Bensoussan, J. Pharm. Biomed. 47 (2008) 494. 